\title{
Candida endophthalmitis in a heroin addict: a case report
}

\author{
J, F, SALMON, ${ }^{1}$ BETTY M. PARTRIDGE, ${ }^{2}$ AND D. J. SPALTON ${ }^{1}$ \\ From the ${ }^{1}$ Ophthalmology and ${ }^{2}$ Microbiology Departments, Charing Cross Hospital Medical School, \\ London W6 8RF
}

SUMMARY Metastatic candida endophthalmitis presented as a unilateral panuveitis in an apparently healthy drug addict. Diagnosis was confirmed by vitrectomy. The eye made an excellent response to treatment with the new antifungal drug, ketoconazole, and 5-fluorocytosine. Problems raised in the diagnosis and management of this infection are discussed.

Candida, yeast-like fungi, are frequent commensals of the human mouth and gastrointestinal tract, but may give rise to systemic infection in the debilitated patient under treatment or in narcotic addicts using self-administered unsterile injections. ${ }^{1-6}$ Candida endophthalmitis is being recognised in these groups with increasing frequency, but the diagnosis is often difficult to establish. We describe a case of uniocular Candida albicans endophthalmitis in a young heroin addict which illustrates these difficulties and the concomitant problems in the choice of antifungal therapy in treatment.

\section{Case report}

A 21-year-old male presented with a painful red eye and decreased vision which had been present for 2 weeks. On examination he was otherwise well. The visual acuities were $6 / 36$ right and 6/6 left. He had a right acute anterior uveitis with inflammatory cells seen in both the aqueous and anterior vitreous gel. The left eye was entirely normal. Routine investigations included a full blood count, which was normal; the erythrocyte sediment rate was $8 \mathrm{~mm} / \mathrm{h}$; chest and sacroiliac joint $x$-rays were normal; tests for syphilis were negative, and a toxoplasmosis titre was not significant.

Treatment was begun with topical corticosteroid and atropine drops. He was followed up at regular intervals over the next 2 weeks, and although his visual acuity remained unchanged there appeared to be no response to initial treatment. One week later the visual acuity fell to $6 / 60$ in the right eye, and a fluffy white vitreous opacity was observed retro-

Correspondence to D. J. Spalton, Ophthalmology Department, Charing Cross Hospital, Fulham Palace Road, London W6 8RF. lentally with considerable vitreous cellular infiltrate (Fig. 1). The patient was given prednisolone (25 $\mathrm{mg} / \mathrm{day}$ ) but a week later his vision in this eye had been reduced to counting fingers. The white cottonwool-like ball could be seen in the vitreous, but no retinal details could be observed. At this stage, it was established that he had been taking intravenous heroin for the previous 12 months. A presumptive diagnosis of fungal endophthalmitis was made, and he was admitted to hospital.

Further investigations included liver function tests, which were normal. Hepatitis B antigen test was negative, but tests for hepatitis B (core and surface) antibodies were positive, indicating previous infection

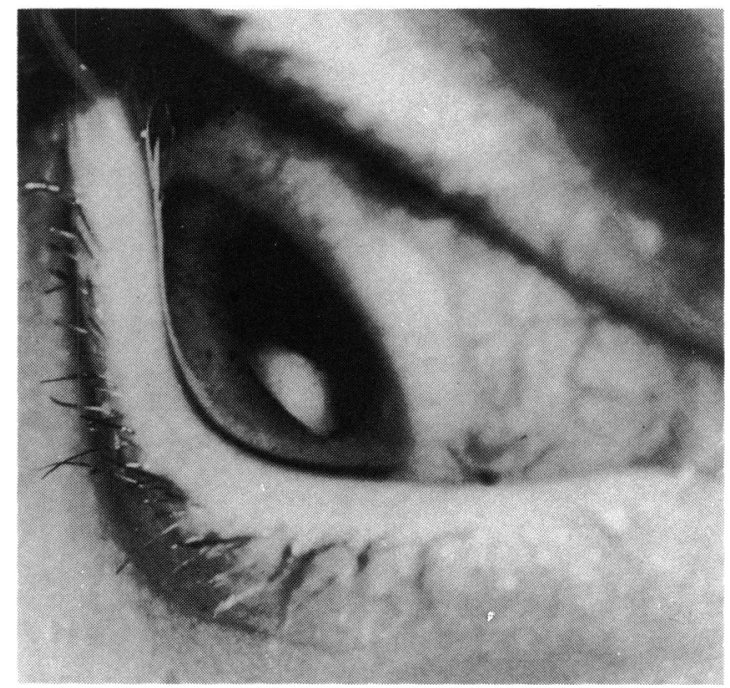

Fig. 1 Right eye showing a white retrolental mass which was removed by pars plana vitrectomy. 
with hepatitis B virus. There was no evidence of yeasts or other fungi in blood or urine. Serum precipitin tests for candida and aspergillus were also negative, but an agglutinating titre of $1 / 32$ against $C$. albicans was recorded. A diagnostic pars plana vitrectomy was performed, the central gel removed, and spun deposits examined: Gram-stained films showed only inflammatory cells and no organisms. No bacteria or fungi were isolated from cultures on blood and peptone-dextrose agar. No candida antigen was detected in the supernatant vitreous fluid.

A further vitrectomy was performed one week later as the eye continued to deteriorate. At this operation as much of the white retrolental mass was aspirated as possible under direct vision. In this specimen a few budding yeasts with occasional short pseudohyphae were seen in the Gram-stained tissue, and cultures produced pure growth of Candida albicans. ${ }^{7}$ These isolates were fully sensitive to antifungal drugs (amphotericin B, 5-fluorocytosine, miconazole, and ketoconazole) with minimum inhibitory concentrations of $0.5 \mu \mathrm{g} / \mathrm{ml}$ or less after 24 hours' incubation.

Treatment was begun with 5-fluorocytosine (150 $\mathrm{mg} /$ day in 3 doses) and miconazole $(1800 \mathrm{mg} / \mathrm{day}$ intravenously in divided doses). The miconazole was discontinued after one day because of severe vomiting, stomach cramps, and dystonia, probably produced by concomitant phenothiazines given for these symptoms. The new antifungal drug, ketoconazole $(200 \mathrm{mg} / \mathrm{kg} /$ day oral administration) was substituted for miconazole, and, apart from a minor

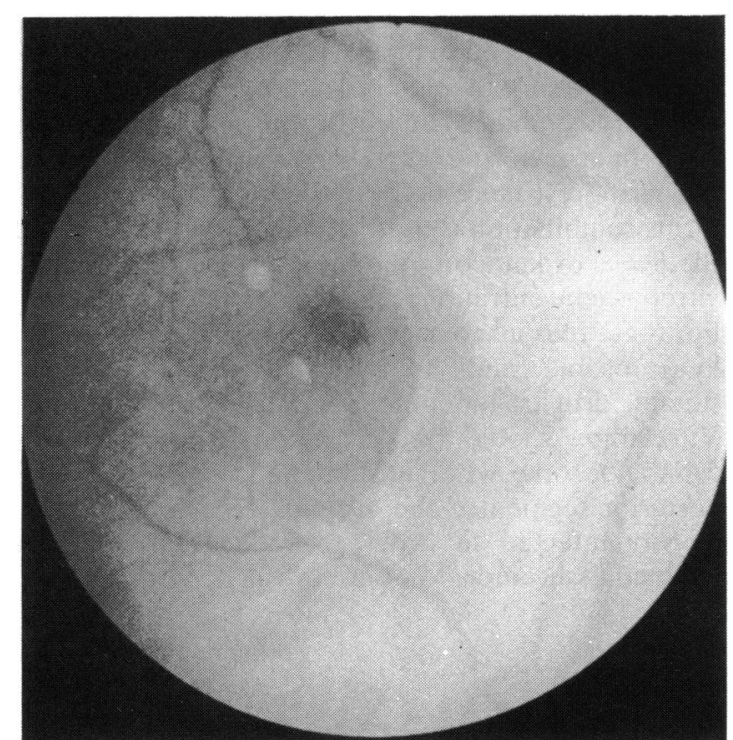

Fig. 2 Right eye following resolution of the infection. $A$ preretinal membrane extending from the area of the peripheral lesion can be seen distorting the macula. transient rise in serum transaminases and alkaline phosphatase, the patient made an excellent response. The eye quietened and vision improved to finger counting, with macula traction from a large retinal traction band passing from the site of the lesion to the optic disc (Fig. 2). Antifungal treatment was discontinued after 3 weeks, and at this stage, just prior to discharge, the retina detached. This was treated by conventional encirclement and plombage with anatomical success. The patient was discharged from hospital with an acuity of finger counting and no signs of active fungal disease.

Further examination of the eye 3 months later showed no change. The serum produced a weak precipitin reaction against $C$. albicans and a 2 -fold rise in agglutinating titre, indicating an immune response to the infection.

\section{Discussion}

Candida endophthalmitis appears from published reports to be much less common in Great Britain than it is in North America, though it is being reported with increasing frequency. The disease seems to affect either immunocompromised and seriously ill patients on high doses of antibiotics or corticosteroids with long-term indwelling intravenous catheters, or intravenous drug addicts.

Many of the patients in the first group develop widespread candidiasis, and in these patients a presumptive ocular diagnosis can be made by growing the yeast from a wide variety of sources, such as blood, urine, or sputum, on the assumption that ocular i.ffection is due to the same species. However. drug addicts present a different and more difficult problem. It is noticeable from previous reports ${ }^{2-5}$ and in our case that the majority of these patients appear generally well and without evidence of disseminated candidiasis; blood and urine cultures may be negative and serological tests for candida either equivocal or negative.

Ocular involvement occurs through haematogenous spread of the yeast, and in the drug addict probably follows an intravenous infection rather than persorption from the gut. Why the eye should be specially liable to infection is not clear, but longstanding vascular endothelial damage can be produced by a previous uveitis ${ }^{8}$ and may explain the ocular sequestration of the yeast. Experimentally, the yeast enters the eye from a chorioretinal focus which bursts into the vitreous, where it proliferates protected by the blood ocular barriers. ${ }^{19}$ In man these foci can be seen as small fluffy white areas on the retinal surface, and when recognised clinically early in the course of the disease they are most helpful in making a presumptive diagnosis. But frequently, and 
especially in drug addicts, the diagnosis of fungal endophthalmitis is not considered until the vitreous is opaque and no retinal detail can be seen. In these circumstances a fungal endophthalmitis must be considered a strong possibility, especially if a posterior uveitis does not respond to steroid therapy or if focal accumulations, sometimes described as a 'string of pearls', can be seen within the vitreous. The disease carries an extremely poor visual prognosis unless the aetiology can be established and early treatment with an antifungal agent initiated.

Confirmation of a presumptive diagnosis of fungal endophthalmitis usually implies a pars plana vitrectomy. This procedure has the theoretical therapeutic advantage of decreasing the infective load within the eye, and removing the vitreous scaffold to allow better and more rapid penetration of antifungal agents into the eye, but against this must be set the well known risks of the operation. ${ }^{10}$ Since patients frequently carry a variety of yeasts. ${ }^{11}$ culture from the ocular tissue is the only sure method of establishing the aetiology of the endophthalmitis.

In the light of our case and others the technique of obtaining and processing the vitrectomy specimen appears to be of the greatest importance. Recovery of the yeast from the vitreous gel is difficult, since it appears to be localised by necrotic inflammatory cells. ${ }^{3}$ It is therefore essential to remove a focal lesion if one is present. ${ }^{10}$ This would account for our failure to isolate the yeast from the first specimen. After the second operation Candida albicans was readily isolated from the tissue.

The ideal antifungal therapy for candida endophthalmitis has yet to be defined, though systemic treatment is the most satisfactory. Until recently the polyene macrolide, amphotericin $B$, has been the mainstay of treatment despite the risk of toxic side effects. ${ }^{12}$ It does not penetrate the uninflamed eye, and it is difficult to obtain adequate therapeutic levels in the vitreous by systemic administration. ${ }^{9}$ Nevertheless it has been used with frequent success in the treatment of $C$. albicans endophthalmitis. Some authors have recommended that intraocular amphotericin B should be given at the time of operation, ${ }^{13}$ but the drug is toxic to the eye, and with the advent of more potent antifungal agents with good ocular penetration this no longer seems to be necessary.

Unlike amphotericin B, 5-fluorocytosine (flucytosine), a fluorinated pyrimidine, is readily absorbed from the gut. It has relatively few toxic effects, and good penetration into the eye. ${ }^{14} \mathrm{~A}$ vitreous concentration of $60 \mu \mathrm{g} / \mathrm{ml}$ of the drug in a patient with a serum level of $70 \mu \mathrm{g} / \mathrm{ml}$ has been recorded (Hay RJ, personal communication). Unfortunately, even if the yeast is initially susceptible to the drug, as our isolate of C. albicans was, resistance may develop fairly rapidly during therapy, and it is considered advisable to use another drug in combination. There is some evidence of synergy with amphotericin B, though this drug may enhance the toxic effect of 5-fluorocytosine on the bone marrow, causing thrombocytopenia and neutropenia. ${ }^{15}$

The imidazole derivatives, which include miconazole and ketoconazole, have a broad spectrum of antifungal activity and low toxicity. Like amphotericin B they alter the permeability of the cell membrane, inhibiting its synthesis and suppressing proliferation and development of the pseudohyphae of $C$. albicans. ${ }^{16}$ Although candida is sensitive to miconazole, the drug usually has to be given intravenously because of poor absorption from the gut. Doses as high as $2400 \mathrm{mg} /$ day may be necessary, and at this dosage distressing side effects such as nausea and vomiting may be encountered.

Ketoconazole, a new antifungal preparation, is considered a major advance in the treatment of fungal infections. It has the advantage of being watersoluble at low $\mathrm{pH}$ and therefore of being readily absorbed from the gut. ${ }^{17}$ Therapeutic levels of the drug are rapidly obtained after a single oral dose and are maintained for several hours. Distribution studies suggest that moderate tissue levels are obtained in most internal organs except the brain. Ketoconazole can penetrate into the cerebrospinal fluid, but detectable concentrations have been observed only in patients with inflamed meninges. ${ }^{18}$ Although ketoconazole has limited toxicity, a transient rise in liver enzymes has been observed in some patients. including our own, and hepatitis has been described as a rare complication of its use. ${ }^{19}$

In our patient there appeared to be no side effects from the combined administration of ketoconazole and 5-fluorocytosine over a period of 3 weeks, and the danger of resistance to 5-fluorocytosine is minimised if this combination is used. It may also be a risk that the levels of ketoconazole do not reach an effective vitreous concentration if ketoconazole is used alone. For these reasons we suggest that the combination of ketoconazole and 5-fluorocytosine is the most suitable drug treatment for candida endophthalmitis. Vitrectomy should be performed if the diagnosis cannot be otherwise substantiated, or to clear the posterior segment if the vitreous gel is opaque and heavily infected in order to reduce the bulk of infected tissue and allow better drug penetration.

\footnotetext{
References

1 Edwards JE. Foos RY. Montgomerie JZ. Guze LB. Ocular manifestations of candida septicaemia: review of seventy-six cases of hematogenous candida endophthalmitis. Medicine (Baltimore) 1974; 53: 47-75.
} 
2 Getnick RA. Rodrigues MM. Endogenous fungal endophthalmitis in a drug addict. Am J Ophthalmol 1974; 77: 680-3.

3 Aguilar GL. Blumenkrantz MS. Egbert PR. McCulley JP. Candida endophthalmitis after intravenous drug abuse. Arch Ophthalmol 1979; 97: 96-100.

4 Elliott JH. O'Day DM. Gutow GS. Podgorski SF. Akrabawi MS. Mycotic endophthalmitis in drug abusers. Am J Ophthalmol 1979; 88: $66-72$.

5 Blumenkrantz MS. Stevens DA. Therapy of endogenous fungal endophthalmitis: miconazole or amphotericin B for coccidioidal and candidal infection. Arch Ophthalmol 1980; 98: 1216-20.

6 Vickers S. Graham E. Spalton DJ. Candida endophthalmitis presenting as bilateral posterior uveitis. $J R$ Soc Med in press.

7 Denny MJ. Partridge BM. Tetrazolium medium as an aid in the routine diagnosis of Candida. J Clin Pathol 1968; 21: 383-6.

8 Howes EL. Cruse VK. The structural basis of altered vascular permeability following intraocular inflammation. Arch Ophthalmol 1978; 96: 1668-76.

9 Jones DB. Chemotherapy of experimental endogenous Candida albicans endophthalmitis. Trans Am Ophthalmol Soc 1980; 78: 846-95.

10 Snip RC. Michels RG. Pars plana vitrectomy in the management of endogenous candida endophthalmitis. Am J Ophthalmol 1976; 82: $699-704$
11 Fitzsimons RB, Nicholls MD, Billson FA, Robertson TI, Hersey P. Fungal retinitis: a case of Torulopsis glabrata infection treated with miconazole. Br J Ophthalmol 1980; 64: 672-5.

12 Cohen J. Antifungal therapy. Lancet 1982; ii: 532-7.

13 Axelrod AJ, Peyman GA. Intravitreal amphotericin B treatment of experimental fungal endophthalmitis. Am J Ophthalmol 1973; 76: 584-8.

14 Walsh A. Haft DA, Miller MH. Loran MR. Freidman AH. Ocular penetration of 5-fluorocytosine. Invest Ophthalmol Visual Sci 1978; 17: 691-4.

15 Medoff G. Kobayashi GS. Strategies in the treatment of systemic fungal infections. $N$ Engl J Med 1980; 302: 145-55.

16 Borgers M. Mechanism of action of antifungal drugs, with special reference to the imidazole derivatives. Rev Infect Dis 1980; 2: 520-34.

17 Van der Meer JWM, Keuning JJ. Scheijgrond HW, Heykants J, Van Cutsem J, Brugmans $J$. The influence of gastric acidity on the bioavailability of ketoconazole. J Antimicrob Chemother 1980; 6: $552-4$.

18 Brass C, Galgiani JN, Blaschke TF, Defelice R, O'Reilly RA, Stevens DA. Disposition of ketoconazole, an oral antifungal, in humans. Antimicrob Agents Chemother 1982; 21: 151-8.

19 Heiberg JK. Svejgaard E. Toxic hepatitis during ketoconazole treatment. Br Med J 1981; 283: 825-6. 
and sources of vitamin $A$, and the precipitating effects of measles and diarrhoea on xerophthalmia. Corneal xerosis and ulceration are associated with severe protein-energy malnutrition and precipitating illnesses and show a peak incidence between 6 months and 3 years, while conjunctival xerosis does not have these associations and has a peak incidence of 3 to 6 years.

The longest section of the book deals with the formulation of effective intervention programmes. Dr Sommer suggests that an outside expert may make an important contribution to this process by visiting and making records at selected sites and interacting with local public health and medical personnel and nutritionists. Such preliminary studies may permit a rational approach to the mounting of complex, expensive, and time-consuming prevalence surveys. Dr Sommer lays down objective, clinical, and biochemical parameters for the diagnosis of xerophthalmia, and outlines the identification of populations at risk, sample size, and stratification of sampling, as well as the personnel involved for such studies, the data to be collected, and its subsequent analysis.

The final sections deal with treatment and prevention. Oral retinol palmitate or acetate $(200000 \mathrm{IU})$ is indicated immediately on diagnosis and repeated on the following day. Additional doses are given 1 to 2 weeks later and more frequently in severe protein-energy malnutrition until the protein status improves. Where oral treatment is impracticable, the parenteral route is used giving the watermiscible retinol palmitate (100000 IU). This treatment is combined with the management of dietary deficiency and intercurrent illness. Indeed recurrence can be prevented only if mothers can modify diet to increase the content of vitamin A from inexpensive sources, such as mango, papaya, carrots, and dark-green leafy vegetables. Some emphasis is rightly laid on re-education of communities at risk in the use of foodstuffs, often abundant sources of provitamin A, based on a knowledge of local conditions. The problems of periodic dosing schedules of either infants at risk or potential mothers and of fortification programmes are also discussed.

This publication is already no doubt familiar to nutritionists and public health workers concerned with Third World countries. Every ophthalmologist should take the opportunity of reading it and it will no doubt stimulate many ophthalmologists in training to interest themselves in the possibilities of preventive ophthalmology. The guide is well printed and lavishly illustrated with coloured clinical photographs of the various stages of vitamin A deficiency. This is a particularly valuable feature which will no doubt be of great help to field workers.

\section{Notes}

\section{Genetics meeting in Ghent}

The International Society for Genetic Eye Diseases will hold the 1984 meeting in Ghent, Belgium, on 12-13 May under the auspices of the University of Ghent. Topics include: hereditary optic nerve diseases, mandibulofacial dysostoses, corneal and anterior chamber anomalies, and retinitis pigmentosa, gyrate atrophy, vitreoretinal degenerations. Further details from Professor Jules François, Graaf de Smet de Naeyerplein 15, B9000, Ghent, Belgium.

\section{European Strabismological Association}

The 14th meeting of the European Strabismological Association. (ESA) will take place in Copenhagen 18-20 May 1984 as a satellite congress to the VIIth Congress of the European Society of Ophthalmology in Helsinki on 21-25 May 1984. The main topic will be: mechanical aspects influencing squint surgery. Free papers will be accepted. Further details from the ESA Congress Office, Eye Department, Rigshospitalet, 9 Blegdamsvej, DK-2100 Copenhagen, Denmark.

\section{Abstracts from $B J O$}

The Commonwealth Mycological Institute announces that papers published in the $B J O$ are regularly abstracted in the Review of Medical and Veterinary Mycology.

\section{Neuro-ophthalmology meeting}

The 6th Meeting of the International Society of Neuroophthalmology and the 7th Congress of the Study Group of Neuro-ophthalmology and Neurogenetics of the World Federation of Neurology will hold a joint meeting in Antwerp, Belgium, on 14-18 May 1984. Details from Professor A. Neetens, Academic Hospital, University of Antwerp, Wilrijkstraat 10, 2520 Edegem, Belgium.

\section{Correction}

In the paper entitled 'Candida endophthalmitis in a heroin addict: a case report,' by J. F. Salmon, Betty M. Partridge, and D. J. Spalton (BJO 1983; 67: 306-9) the dose for 5 -fluorocytosine should have been given as $150 \mathrm{mg} / \mathrm{kg} /$ day instead of $150 \mathrm{mg} /$ day, and the dose for ketoconazole should have been given as $200 \mathrm{mg} /$ day instead of $200 \mathrm{mg} / \mathrm{kg} / \mathrm{day}$. 